\title{
Two-photon fluorescence microscope with a hollow-core photonic crystal fiber
}

\author{
Shih-Peng Tai, Ming-Che Chan, Tsung-Han Tsai, Shi-Hao Guol, Li-Jin Chen, and Chi- \\ Kuang Sun* \\ Department of Electrical Engineering and Graduate Institute of Electro-Optical Engineering, National Taiwan \\ University, Taipei 10617, TAIWAN \\ sun@cc.ee.ntu.edu.tw
}

\begin{abstract}
Self-phase-modulation and group velocity dispersion of near IR femtosecond pulses in fibers restrict their use in two-photon fluorescence microscopy (TPFM). Here we demonstrate a hollow-core photonic crystal fiber based two-photon fluorescence microscope with low nonlinearity and dispersion effects. We use this fiber-based TPFM system to take two-photon fluorescence (chlorophyll) images of mesophyll tissue in the leaf of Rhaphidophora aurea. With less than $2 \mathrm{~mW}$ average power exposure on the leaf at $755 \mathrm{~nm}$, the near zero-dispersion wavelength, chloroplasts distribution inside the mesophyll cells can be identified with a sub-micron spatial resolution. The acquired image quality is comparable to that acquired by the conventional fiber-free TPFM system, due to the negligible temporal pulse broadening effect.
\end{abstract}

(C) 2004 Optical Society of America

OCIS codes: (180.6900) Three-dimensional microscopy; (180.5810) Scanning microscopy.

\section{References and links}

1. W. Denk, J. H. Strickler, and W. W. Webb, “2-Photon Laser Scanning Fluorescence Microscopy,” Science 248, 73-76 (1990).

2. D. Bird and M. Gu, "Resolution improvement in two-photon fluorescence microscopy with a single-mode fiber," Appl. Opt. 41, 1852-1857 (2002).

3. D. Bird and M. Gu, "Fibre-optic two-photon scanning fluorescence microscopy," J. Microscopy, 208, 35-48 (2002).

4. D. Bird and M. Gu, "Compact two-photon fluorescence microscope based on a single-mode fiber coupler," Opt. Lett. 27, 1031-1033 (2002).

5. D. Bird and M. Gu, "Two-photon fluorescence endscopy with a micro-optic scanning head," Opt. Lett. 28, 15521554 (2002)

6. F. Helmchen, D. W. Tank, and W. Denk, "Enhanced two-photon excitation through optical fiber by single-mode propagation in a large core," Applied Optics 41, 2930-2933 (2002).

7. D. G. Ouzounov, K. D. Moll, M. A. Forster, W. R. Zipfel, W. W. Web, and A. L. Gaeta, "Delivery of nanojoule femtosecond pulses through large-core microsrure fibers," Opt. Lett. 27, 1513-1515 (2002).

8. M. E. Fermann, "Single-mode excitation of multimode fibers with ultrashort pulses," Opt. Lett. 23, $52-54$ (1998).

9. R. F. Cregan, B. J. Mangan, J. C. Knight, T. A. Birks, P. St. J. Russell, P. J. Roberts, and D. C. Allan, "Singlemode photonic band gap guidance of light in air," Science 285, 1537-1539 (1999).

10. C. J. S. de Matos and J. R. Taylor, T. P. Hansen, K. P. Hansen, and J. Broeng, ”All-fiber chirped pulse amplification using highly-dispersive air-core photonic bandgap fiber," Opt. Express 11, 2832-2837 (2003) http://www.opticsexpress.org/abstract.cfm?URI=OPEX-11-22-2832

11. J. Limpert, T. Schreiber, S. Nolte, H. Zellmer, and A. Tünnermann, ”All fiber chirped-pulse amplification system based on compression in air-guiding photonic bandgap fiber," Opt. Express 11, 3332-3337 (2003) http://www.opticsexpress.org/abstract.cfm?URI=OPEX-11-24-3332

12. D. G. Ouzounov, F. R. Ahmad, D. Muller, N. Venkataraman, M. T. Gallagher, M. G. Thomas, J. Silcox, K. W. Koch, and A. L. Gaeta," Generation of Megawatt Optical Solitons in Hollow-Core Photonic Band-Gap Fibers" Science 301, 1702-1704 (2003)

13. G. Bouwmans, F. Luan, J. C. Knight, P. St. J. Russel, L. Farr, B. J. Mangan, and H. Sabert, "Properties of a hollow-core photonic bandgap fiber at 850nm wavelength," Opt. Express 11, 1613-1620 (2003) http://www.opticsexpress.org/abstract.cfm?URI=OPEX-11-14-1613

14. W. Gobel, A. Nimmerjahn, and F. Helmchen, "Distortion-free delivery of nanojoule femtosecond pulses from a Ti:sapphire laser through a hollow-core photonic crystal fiber," Opt. Lett. 29, 1285-1287 (2004).

15. http://www.crystal-fibre.com 
16. U. K. Tirlapur, and K. Konig, "Femtosecond near-infrared lasers as a novel tool for non-invasive real-time highresolution time-lapse imaging of chloroplast division in living bundle sheath cells of Arabidopsis," Planta 214, 110 (2001).

17. I-H. Chen, S.-W. Chu, C.-K. Sun, P. C. Cheng, and B.-L. Lin, "Wavelength dependent damage in biological multi-photon confocal microscopy: A micro-spectroscopic comparison between femtosecond $\mathrm{Ti}: \mathrm{sapphire}$ and $\mathrm{Cr}$ : forsterite laser sources: A micro-spectroscopic comparison between femtosecond Ti:sapphire and Cr:forsterite laser sources," Opt. Quantum Electron. 34, 1251-1266 (2002).

18. S.-W. Chu, T.-M. Liu, C.-K. Sun, C.-Y. Lin, and H.-J. Tsai, "Real-time second-harmonic-generation microscopy based on a 2-GHz repetition rate Ti:sapphire laser," Opt. Express 11, 933-938 (2003). http://www.opticsexpress.org/abstract.cfm?URI=OPEX-11-8-933

\section{Introduction}

Since the first demonstration in 1990, two-photon fluorescence microscopy (TPFM) has made a great impact on biomedical researches [1]. With its high penetration ability, low out-of-focus photodamage, and intrinsic three-dimensional (3D) sectioning capability, TPFM has been widely applied to various medical diagnosis and genome researches. Recently, optical fibers were introduced into the TPFM systems for remote optical pulse delivery [2-6]. Fiber-based TPFM has advantages including isolating vibrations from lasers and electronic devices, flexible system design, and low crosstalks [2-6]. It is also the first step toward an all-fiber based two-photon fluorescence endoscope. An ideal fiber-based TPFM system should maintain efficient two-photon excitation where high peak power is required. Besides, singlemode propagation is necessary to preserve a spatial profile suitable for diffraction-limited focusing as well as to avoid additional temporal broadening as a result of intermodal dispersion. In previous studies, single-mode optical fibers were introduced into the Ti:sapphire laser based TPFM systems for high energy ultrashort optical pulse delivery [2-6]. However, due to serious temporal broadening [3,6], when femtosecond pulses emitted from a Ti:sapphire laser propagate through the fiber, the two-photon excitation efficiency of the fiberoptic TPFM was much lower than the conventional one. The temporal broadening effect is mainly contributed from group velocity dispersion (GVD) and power dependent nonlinear dispersion (self-phase modulation, SPM) that also leads to significant spectral broadening.

Linear dispersion can be compensated by prechirping pulses with prisms or grating pairs. However, it is difficult to overcome nonlinear broadening effects. Large core fibers have been shown to reduce nonlinear effects, but temporal broadening still occurred at high intensities [6,7]. In addition, larger cores usually support propagation of several transverse modes and thus decrease the spatial resolution of TPFM and induce temporal pulse broadening due to intermodal dispersion. For a limited transmission distance about several meters this conflict can be solved [6-8], but still limits long distance TPFM application. Moreover, in these studies, prefiber dispersion compensation were still required [6,7].

To solve these difficulties, pulses propagating in a hollow air core fiber is desired to minimize dispersion and nonlinear SPM effects. In 1999, air-guided fibers using photonic bandgap structures have been developed [9]. In this fiber, light can be guided in an air core due to so-called Bragg photonic bandgap guidance. Such kind of single-mode air-guidance photonic crystal fiber (PCF) has been used as a pulse compressor in all-fiber chirped pulse amplification systems due to its large anomalous waveguide dispersion $[10,11]$ at specific wavelengths. More importantly, the low nonlinear SPM effect in air makes these hollow-core PCFs suitable for high energy ultrashort pulses delivery [12-14]. Recent studies in $~ 800 \mathrm{~nm}$ wavelength region strongly support the fact that hollow-core PCFs are suitable for TPFM applications $[13,14]$.

Here we demonstrate a hollow-core PCF-based TPFM system at a central wavelength of 755nm with a femtosecond Ti:sapphire laser. By replacing the conventional single-mode fiber with the hollow-core PCF, much improvement of two-photon fluorescence excitation efficiency and image quality are achieved due to negligible nonlinear SPM and dispersion 
effects at the near-zero dispersion wavelength of this hollow-core PCF.

\section{Material and methods}

The experiment setup of the TPFM with a hollow-core PCF is shown in Fig. 1. 200fs pulses with a $82 \mathrm{MHz}$ repetition rate generated from a Ti:sapphire laser (Spectra Physics, Tsunami) were coupled by an 20X objective (Olympus LMPlan/IR, 0.4NA) into a 1-m-long hollowcore PCF (AIR-6-800, Crystral Fibre A/S) with a $6 \mu \mathrm{m}$ core diameter surrounded with a $\sim 122 \mu \mathrm{m}$ diameter cladding layer [15]. The transmission loss of this fiber is below $0.4 \mathrm{db} / \mathrm{m}$ from $750 \mathrm{~nm}$ to $800 \mathrm{~nm}$. A variable neutral density filter was placed before the coupling objective to control laser input power. At the fiber output, a 10X objective (Olympus UMPlanFI, 0.3NA) was used as a beam collimator. Two flipped mirrors were used to control laser direction into a home-built optical autocorrelator, a spectrum analyzer, or a confocal scanning microscope (Olympus FV300 scanning unit combined with IX71 inverted microscope). The home-built optical autocorrelator was used to measure the temporal profile of the ultrashort optical pulses. Inside the autocorrelator, the ultrashort optical pulse was divided into two equal pulses by a beam splitter. One pulse passed through a fixed delay line and was chopped at $1 \mathrm{KHz}$ by a mechanical chopper while the other passed through a computer controlled variable delay line (Newport UTM25PP.1) with a $0.1 \mu \mathrm{m}$ step size. The dynamic range of the delay stage is $25 \mathrm{~mm}$ corresponding to $\sim 167 \mathrm{ps}$ time delay. A lens with $31 \mathrm{~mm}$ focal length (Special Optics 54-17-30-700-900) was used to focus both laser beams into a $300-\mu \mathrm{m}$-thick $\beta$-BBO crystal with a $>16 \mathrm{~nm}$ phase matching bandwidth in $750 \mathrm{~nm}$ $800 \mathrm{~nm}$. The SHG autocorrelation second-harmonic-generation signals were then detected by a silicon-based p-i-n photodetector (THORLABS, DET100) connected into a lock-in amplifier (Stanford Research Systems SR830). The biological sample used to study imaging performance of this hollow-core photonic crystal fiber based two-photon fluorescence microscopy is the leaf of Rhaphidophora aurea, and a water immersion objective (Olympus, LUMPlanFI/IR, 0.9NA) was used to focus laser beam into the sample. To guarantee the signals we collected are two photon fluorescence of chlorophyll, a short wave pass color filter (Lambda Research Optics SWP-1202U-700) and a 40nm bandwidth interference filter (Lambda Optics 670-F40-12.7), corresponding to the 670nm fluorescence peak of chlorophyll [16], were inserted before the photo multiplication tube in the Olympus FV300 scanning unit.

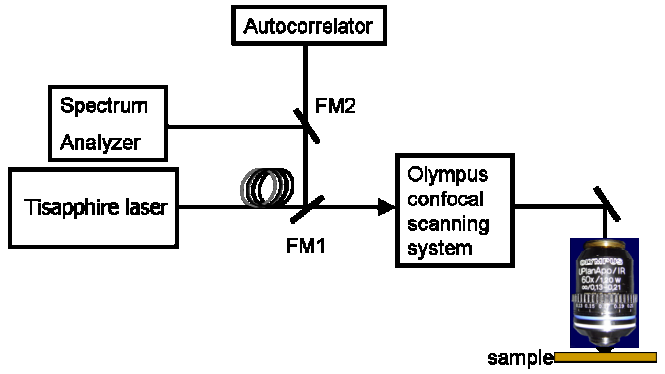

Fig. 1 Experiment setup of the TPFM with a hollow-core photonic crystal fiber.

\section{Results and discussion}

\subsection{Property of the hollow core PCF: self-phase modulation effect}

At first, we measured the spectra of the input and output pulses before and after the PCF for different central wavelengths at different average output powers from $20 \mathrm{~mW}$ to $120 \mathrm{~mW}$. The power dependent spectra were analyzed from $750 \mathrm{~nm}$ to $800 \mathrm{~nm}$. The spectral full-width-halfmaximum (FWHM) bandwidth of the input pulses was controlled to be $\sim 10 \mathrm{~nm}$. Figure 2 shows examples of the input and fiber-output spectra measured at 750nm, 755nm, 780nm, and $795 \mathrm{~nm}$, respectively. No power dependency on the output spectra can be observed, 
demonstrating negligible nonlinear SPM effects even at $120 \mathrm{~mW}$ average output power which corresponds to pulse energies of $1.5 \mathrm{~nJ}$ transmitted through the PCF. Due to limited transmission bandwidth between $750 \mathrm{~nm}-800 \mathrm{~nm}$, PCF output intensity decays quickly below 750nm, as observed in Fig. 2(a) and Fig. 2(b).
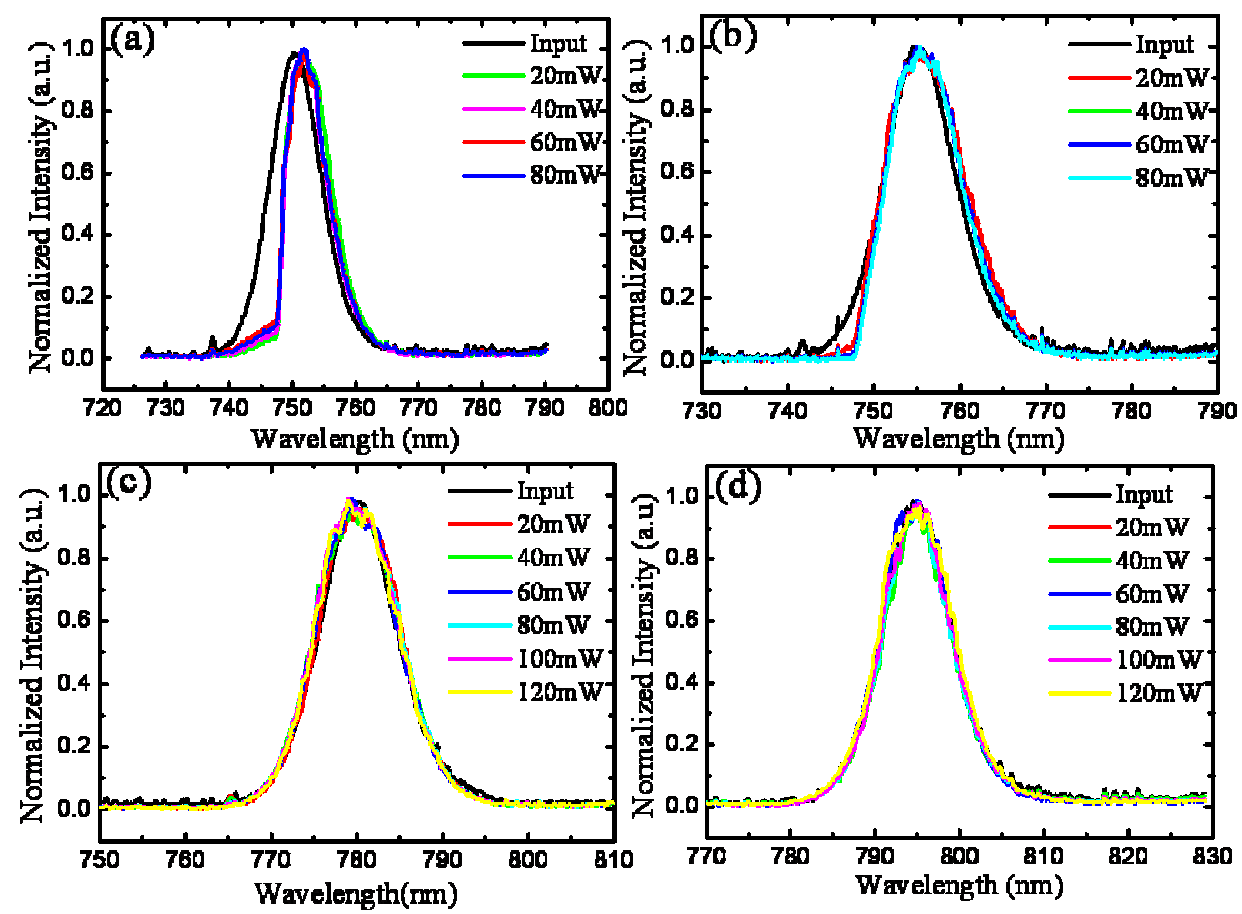

Fig. 2 Power dependent output spectra of the hollow-core PCF at central wavelengths of (a) $750 \mathrm{~nm}$, (b) $755 \mathrm{~nm}$, (c) $780 \mathrm{~nm}$, and (d) 795nm. Input spectra are also provided for comparison.

\subsection{Property of the hollow core PCF: group velocity dispersion}

Group velocity dispersion (GVD) characteristic of the guided mode through the low-loss region in the hollow core PCF was studied by measuring the output pulsewidth as a function of wavelength. We used the home-built optical autocorrelator to measure the input and output pulsewidths from $750 \mathrm{~nm}-790 \mathrm{~nm}$ at $80 \mathrm{~mW}$ and $120 \mathrm{~mW}$ average output power levels, corresponding to pulse energies of $1 \mathrm{~nJ}$ and $1.5 \mathrm{~nJ}$. The input pulsewidth was $\sim 200 \mathrm{fs}$ with a $\operatorname{sech}^{2}$ pulse shape. Figures 3(a-d) show examples of the measured intensity autocorrelation traces with $80 \mathrm{~mW}$ average transmitted power. The relation between pulsewidth and central wavelength measured at $80 \mathrm{~mW}$ and $120 \mathrm{~mW}$ average output power levels are shown in Fig. 3(e). The widths of the measured autocorrelation traces at different central wavelengths were found to be independent of input power, as shown in Fig. 3(e). Due to a strong GVD effect, the pulsewidths at $780 \mathrm{~nm}$ and $790 \mathrm{~nm}$ were found to be stretched up to $4.9 \mathrm{ps}$ and $12.4 \mathrm{ps}$ (based on $\operatorname{sech}^{2}$ pulseshape assumption), corresponding to GVD values of 390ps/ $/ \mathrm{km} / \mathrm{nm}$ and $1060 \mathrm{ps} / \mathrm{km} / \mathrm{nm}$, agreeing with a previous measurement [15]. From Fig. 3, zero-dispersion wavelength can be observed to be $\sim 755 \mathrm{~nm}$. The output pulsewidth at $755 \mathrm{~nm}$ was $250 \mathrm{fs}$ at $80 \mathrm{~mW}$ (based on $\operatorname{sech}^{2}$ pulseshape assumption). Compared with the 200fs input pulsewidth, the temporal broadening ratio, defined as output pulsewidth divided by input pulse width, is only 1.25 which is much better than the results obtained in standard single mode fibers $[3,6]$. This weak temporal broadening is possibly due to high order dispersion, the reduced spectral 
width, and the residue nonlinear effect due to wave function extension into the non-air cladding region.
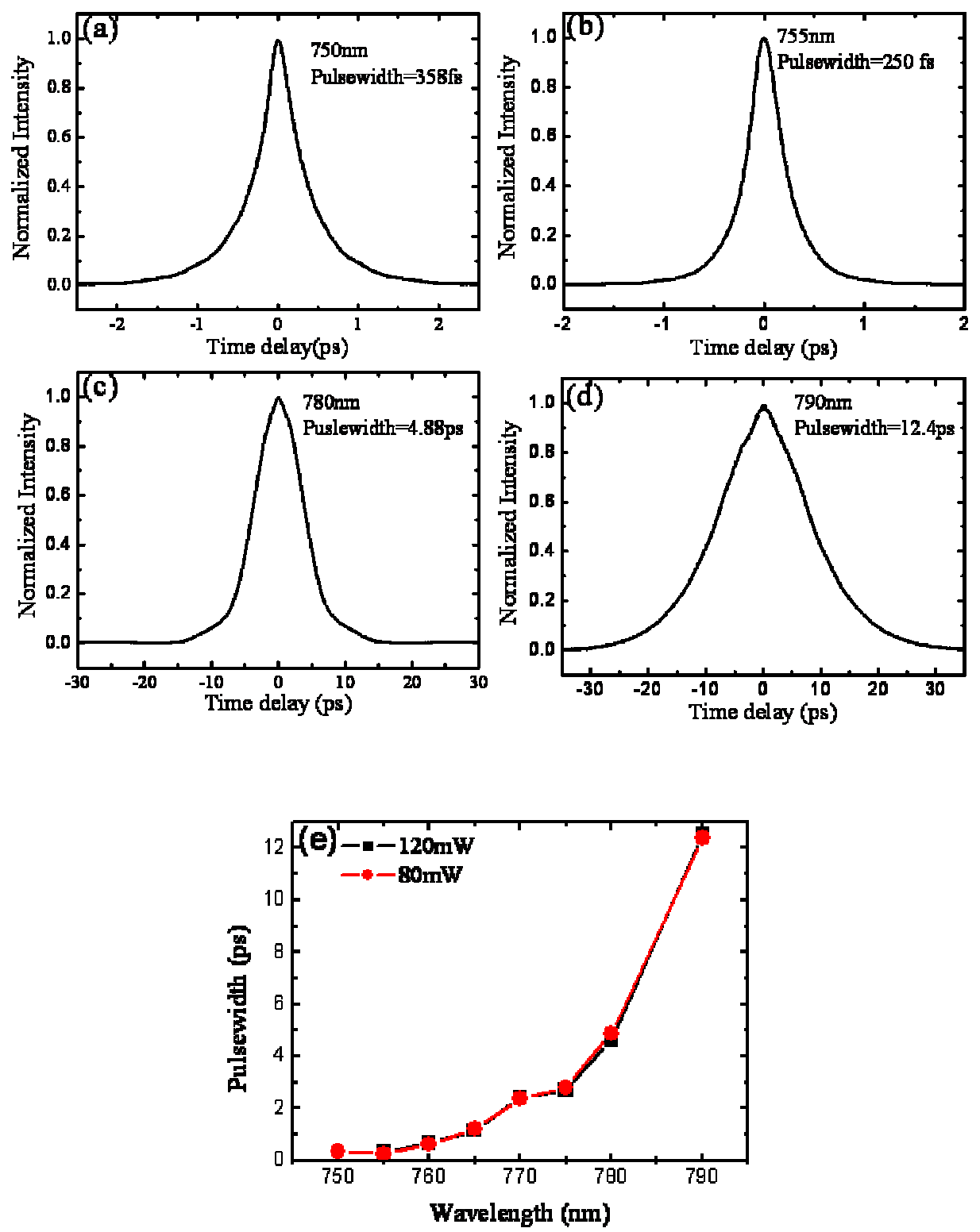

Fig. 3 (a)-(d) Intensity autocorrelation trace with $80 \mathrm{~mW}$ average power transmitted through the hollow core PCF at different central wavelengths. (e) The relation between temporal pulse width and center wavelength at $80 \mathrm{~mW}$ and $120 \mathrm{~mW}$ average power transmitted through the hollow core PCF.

\subsection{Hollow-core photonic crystal fiber based two-photon fluorescence microscopy}

Due to nearly distortion-free propagation of high energy femtosecond pulses $(\sim 755 \mathrm{~nm}$ in our specific case) in the hollow-core PCF, it has important implications for applications on 
nonlinear optical microscopy. Here, we study the wavelength dependent imaging performance of the constructed hollow-core PCF-based two-photon fluorescence microscopy system. To ensure the image we took was really from the two-photon fluorescence of chlorophyll, we measured the relation between the collected fluorescence power and the excitation power for $750 \mathrm{~nm}-790 \mathrm{~nm}$. The measured fluorescence power after the filters was found to be in quadratic dependence on the excitation power over the whole wavelength range we studied, confirming its two-photon nature. An example result measured at $755 \mathrm{~nm}$ is shown as Fig. 4. Figures 5(a-e) shows two-photon fluorescence (chlorophyll) images of the mesophyll tissue in the leaf of Rhaphidophora aurea taken with a conventional fiber-free TPFM system at central wavelengths of $755 \mathrm{~nm}, 760 \mathrm{~nm}, 770 \mathrm{~nm}, 780 \mathrm{~nm}$, and 790nm, respectively. Figure 5 (f-j) shows corresponding chlorophyll images taken with this hollow core PCF-based TPFM system at the central wavelengths corresponding to Figs. 5(a-e). The average illumination powers on the sample were all kept $1.5 \mathrm{~mW}$ to avoid possible photo-damage and photobleaching effects [16-18]. At the near-zero dispersion wavelength $(755 \mathrm{~nm})$, chloroplast distribution inside the mesophyll cells can be identified with a sub-micron resolution (Fig. 5(f)) and the image quality is comparable to that of Fig. 5(a). Although image quality degrades at 760nm and 770nm (Figs. 5(g), 5(h)) due to pulse broadening and thus lowered two photon fluorescence efficiency, chloroplast distributions inside the mesophyll cells can still be clearly observed. Figure 6 shows the TPFM image degradation ratio as a function of wavelength. The image degradation ratio is defined as image intensity acquired with conventional fiber-free TPFM system divided by image intensity acquired with this hollow-core PCF-based TPFM system. Temporal broadening ratio is also provided for comparison. We can observe a strong correlation between both parameters, indicating that image degradation in our hollow-core PCF-based system is dominated by the pulse broadening effect due to waveguide dispersion in the PCF. The strong correlation between both parameters can be observed especially at the near-zero wavelength. At $755 \mathrm{~nm}$ wavelength, the image degradation ratio and temporal broadening ratio are both as low as 1.28 and 1.25 , respectively, indicating negligible temporal broadening and image degradation effects. Comparing with previous single-mode fiber based TPFM system [3], performance of our hollow-core PCF-based TPFM has been greatly improved above ten times. Although the waveguide dispersion of this hollow-core PCF limits this fiber-based TPFM to a restricted tuning range, the linear dispersion induced by the hollow core PCF at wavelength longer than $760 \mathrm{~nm}$ can be compensated by inserting prechirp configurations such as grating pairs or prism pairs. Therefore, with a fixed fiber, the tunable range of this hollow-core PCF-based TPFM can be expected to extend to all low-loss wavelength regions $(750 \mathrm{~nm}-800 \mathrm{~nm})$.

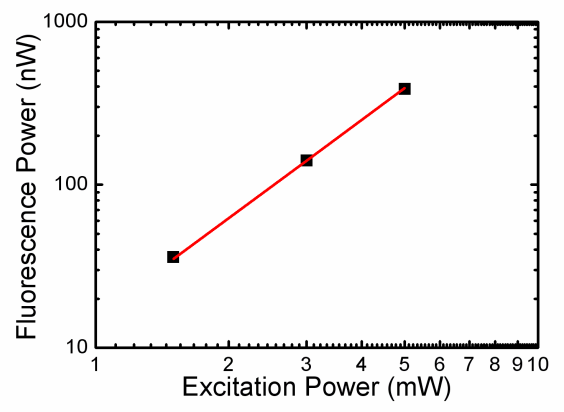

Fig. $4670 \mathrm{~nm}$ fluorescence power (rectangle) vs. $755 \mathrm{~nm}$ excitation power for the mesophyll tissue in the leaf of Rhaphidophora aurea. The well-matched solid line is the slope $=2$ fitting, confirming its two-photon nature 

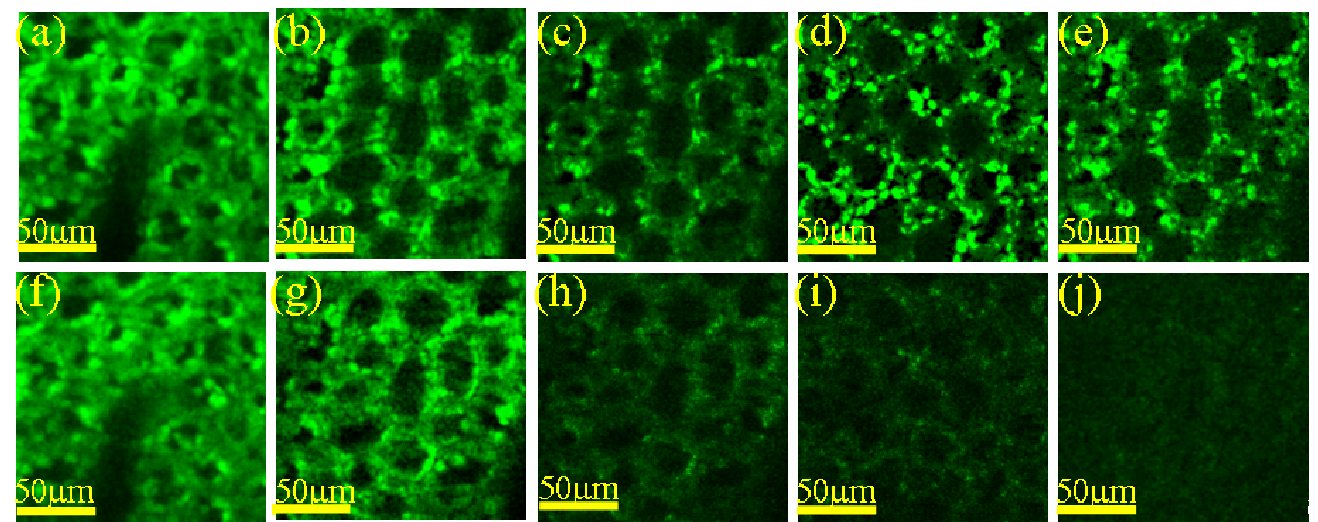

Fig. 5 (a)-(e) Two-photon fluorescence images of mesophyll tissues in the leaf of Rhaphidophora aurea, taken with the conventional fiber-free system. Image size: $160 \mu \mathrm{m} \times$ $160 \mu \mathrm{m}$. (f)-(j) Two-photon fluorescence images of mesophyll tissues in the leaf of Rhaphidophora aurea, taken with the system based on a hollow-core PCF. Image size: $160 \mu \mathrm{m}$ $\times 160 \mu \mathrm{m}$.

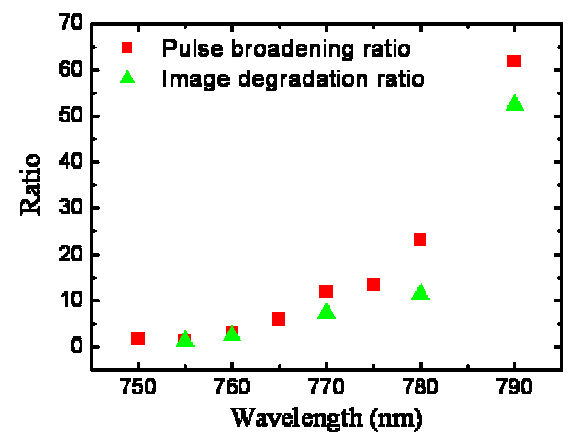

Fig. 6 Wavelength dependent temporal pulse broadening ratio (squares) and two-photon fluorescence image degradation ratio (triangles).

\section{Conclusion}

A fibre-optic TPFM system based on a hollow-core photonic crystal fiber, with low nonlinear SPM effect while high-energy ultrashort pulses passing through the fiber, is demonstrated in this paper. Due to pulse guiding almost exclusively in air, nonlinear SPM effects are minimal with essentially intensity independent propagation over the entire guided wavelength. Much improvement of two-photon fluorescence excitation efficiency and image quality are achieved by minimizing nonlinearity and dispersion at the near-zero dispersion wavelength. Negligible pulse broadening and image degradation effects are demonstrated with this novel hollow-core PCF-based TPFM system.

\section{Acknowledgments}

The authors gratefully acknowledge financial support from National Health Research Institute of Taiwan (NHRI-EX93-9201EI) and National Taiwan University Center for Genomic Medicine. 